\title{
Clinical neuropsychology in the management of myotonic dystrophy
}

\section{Christopher D. Graham PhD DClinPsychol*1, Steven Kemp DClinPsychol, AFBPsS ${ }^{2}$, Ratko Radakovic PhD, $\mathrm{CPsychol}^{3}$, Narinder Kapur PhD, FBPsS ${ }^{4}$}

${ }^{1}$ Leeds Institute of Health Sciences, University of Leeds, Leeds, UK, LS2 9LJ.

${ }^{2}$ Department of Clinical Neuropsychology, Leeds Teaching Hospitals NHS Trust, St. James University Hospital, Leeds, UK, LS9 7TF.

${ }^{3}$ Faculty of Medicine and Health Sciences, Queens Building, University of East Anglia, Norwich, UK, NR4 7TJ

${ }^{4}$ Research Department of Clinical, Educational \& Health Psychology, 1-19 Torrington Place, University College London, WC1E 7HJ

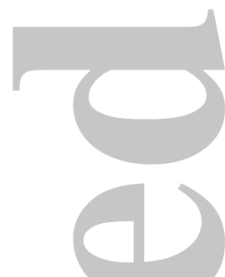

Declaration of Conflicting Interests: The author(s) declared no potential conflicts of interest with respect to the research, authorship, and/or publication of this article

Funding: This work was unfunded.

*Requests for reprints should be addressed to Dr Christopher D. Graham, Leeds Institute of Health Sciences, University of Leeds, Leeds, UK, LS2 9LJ. E-mail: c.d.graham@leeds.ac.uk

Word count main submission: 1898

Word count abstract: 105

Ethical publication statement: "We confirm that we have read the Journal's position on issues involved in ethical publication and affirm that this report is consistent with those guidelines."

This article has been accepted for publication and undergone full peer review but has not been through the copyediting, typesetting, pagination and proofreading process which may lead to differences between this version and the Version of Record. Please cite this article as an 'Accepted Article', doi: 10.1002/mus.26085 
Neuropsychology for DM1

In an earlier edition of Muscle \& Nerve, we outlined how psychological processes interact with biological and social factors to affect quality of life in muscle disorders (1). We then described the use of psychological interventions - focusing mainly on talking therapies - for improving quality of life, mood and general functioning. As in the case of well-cited reviews describing the clinical management of myotonic dystrophy $(2,3)$, neuropsychological assessment and cognitive rehabilitation were not described in detail in our article. The work by Fujino et al. (4), published in this issue of Muscle \& Nerve, suggests that, in the care of patients with myotonic dystrophy (Type 1) at least, the lack of attention to cognitive rehabilitation may have been an oversight. Therefore, in this commentary we discuss the clinical implications of Fujino et al., (4), taking the opportunity to describe the potential for cognitive rehabilitation for myotonic dystrophy.

Fujino and colleagues (4), observed that a large proportion of their participants had quite severe myotonic dystrophy and demonstrated impairment in at least one cognitive domain (i.e. scores at least two standard deviations from population averages). Impairment occurred most frequently in the domains of executive functioning, processing speed, attention, and visuoconstruction. Although this study included no controls for the impact of marked motor impairment, this finding adds to a body of work showing that many people with myotonic dystrophy type 1 experience cognitive difficulties $(5,6)$. Consistent with existing work (7), a significant proportion of participants in the sample recruited by Fujino et al. [4] demonstrated co-morbid behavioural symptoms/changes - with higher levels of apathy and depression apparent in the sample.

The aetiology of cognitive and behavioural symptoms in Fujino et al.'s [4] sample was unclear. Other studies have found an association between unstable cytosine-thymineguanine (CTG) repeat expansion size and cognitive test performance and behaviour changes in myotonic dystrophy $(5,6,8)$. This is unsurprising given that, in myotonic dystrophy type 1 , a positive association exists between brain pathology and CTG repeat expansion (9), while the extent of white matter lesions is positively correlated with cognitive impairment and behavioural symptoms $(10,11)$. In addition, other contextual factors - which may be less dependent on underlying brain pathology - might also affect cognitive functioning. Reactive depression, perhaps resulting from difficulties adjusting to physical symptoms, may worsen 
Neuropsychology for DM1

performance on cognitive testing; fatigue, sleepiness, or pain may also lead to problems with effort.

Importantly, Fujino et al. (4) demonstrated that performance on several cognitive tests was positively correlated with psychosocial aspects of quality of life. The cross-sectional design does not allow us to disentangle the direction of influence between these variables. Yet, this observation does raise the possibility that an improvement in cognitive symptoms, or their impact on functioning, could lead to an improvement in quality of life. Although a caveat is that some reduced insight may be protective of mood (12), cognitive rehabilitation might offer a means to improve outcomes for many people with myotonic dystrophy.

\section{The role of Neuropsychology in the care of myotonic dystrophy}

Cognitive rehabilitation involves two stages: an assessment of cognitive functioning, followed by consequent treatment recommendations. Below, we discuss each stage, with some salient discussion points emerging from Fujino et al. (4).

\section{Characterising a cognitive and behavioural profile}

Cognitive rehabilitation initially entails carrying out a clinical interview and a formal assessment of cognitive functioning - to generate a cognitive and behavioural profile. The clinical interview comprises discussion with the patient and someone who knows them well (e.g. spouse, family member, friend). To estimate expected cognitive functioning, questions are asked about the patient's educational background and developmental history.

Questioning may then switch to examining cognitive failures, behaviour changes, mood and everyday adjustment. This information can be contrasted against estimates of expected functioning to suggest those cognitive domains that are likely to be affected and therefore should be formally assessed.

A formal assessment may then be undertaken, using standardised cognitive tests: cognitive screens, comprehensive test batteries or focal assessment batteries. A cognitive screen (e.g. Repeatable Battery for the Assessment of Neurological Status; RBANS (13)), involving brief tests covering many cognitive domains (e.g. memory, executive functioning, visuospatial functioning, and processing speed), may be administered to assess whether comprehensive 
Neuropsychology for DM1

or focal neuropsychological testing is likely to be of informative. Comprehensive test batteries, such as the Wechsler Adult Intelligence Scale (WAIS) (14), are alternatively or subsequently administered to patients. Such batteries involve detailed testing of many cognitive domains. If indicated, extended testing of a single cognitive domain may also be informative - helping pinpoint more exactly the locations of cognitive impairment. For example, batteries such as the Wechsler Memory Scale (WMS) (15) or the Delis Kaplan Executive Function Scale (DKEFS) (16) can be used to comprehensively assess memory and executive functioning respectively. Raw test scores are then compared to population norms to generate metrics indicating deviations from expected scores, resulting in a cognitive profile. Once a patient's cognitive profile is characterised (domains where they have perform as expected or have strengths, weaknesses and impairments), then information from the clinical interview helps to inform what may explain any findings of impairment.

In light of this information, intervention may take several forms (described in the next sections). Yet there are possible therapeutic benefit of cognitive profile characterisation alone (17). First, in clinic we often note that, for many people, fears about cognitive impairment and excessive attention toward cognitive errors detrimentally affects mood and functioning. Therefore, for those individuals where cognitive impairment was suspected/feared but is not apparent after assessment, cognitive assessment can provide reassurance and reduce pre-occupation with possible cognitive symptoms.

Second, such an assessment might enable a change in perspective for the person with myotonic dystrophy and the system around them - including their clinicians. There is a sizable literature describing aberrant personality profiles in myotonic dystrophy (see(3)). Such a focus on personality may risk the blurring of symptoms (neurocognitive, behavioural symptoms/behaviour or the impact of muscle pathology) with the person who is living with them. Given underlying neuro- and muscle pathology, disease-linked neurocognitive or behavioural changes (e.g. theory of the mind difficulties (18), apathy (4) or other neuropsychiatric features (12)) and/or the impact of living with muscle symptoms (pain, fatigue etc.) may be a more accurate conceptualisation than personality difficulties. If we accept this, then we might more readily see a separation between the person and their symptoms; viewing the person as finding themselves in a difficult context which involves 
Neuropsychology for DM1

living with myotonic dystrophy-caused symptoms (as opposed to being an apathetic person etc.). This may: a) foster greater empathy for the person living with the condition among their clinicians, family and care-givers- as well as self-compassion, and b) in the case of neurocognitive and behavioural symptoms, emphasize that these are not just due to a person's personality but rather are a change or symptom of disease, and may therefore be targets for treatment/clinical research.

In the study by Fijuno et al. (4) a notable limitation was that, in addition to secondary factors such as pain that were outlined above, muscle pathology may have confounded test performance. Several of the cognitive tests employed in their study require timed or accurate physical responses, such as drawing (e.g. the Trail-Making Test or figure copy). It is possible that myotonia and muscle weakness could impede responses - leading to erroneous assumptions of cognitive impairment. Therefore, myotonia and muscle weakness should be considered when selecting neuropsychological tests. Tests that do not require hand movements may be of value. For example, a newer cognitive screen developed for ALS - the Edinburgh Cognitive and Behavioural ALS Screen (19) - has an alternate form requiring verbal response alone. However, speech may also be affected in myotonic dystrophy type 1, and difficulties with articulation could also affect test performance. In addition, test length should also be carefully considered because another confounding factor in assessing performance on cognitive testing can be fatigue or sleepiness (20). Similarly, the impact of muscle symptoms, speech, fatigue or difficulties adjusting to the condition may impact the measurement of behaviour changes. Thus, nuanced or multiple-dimension measures of, for example, apathy (Dimensional Apathy Scale (21)) may be more informative than the singledomain measure used in Fujino et al. [4].

\section{Cognitive rehabilitation}

Although one case study exists (22), we are unaware of any clinical trials of cognitive rehabilitation in myotonic dystrophy. There is a promising literature from trials of cognitive rehabilitation for multiple sclerosis (23) that may be generalizable to myotonic dystrophy. Thus, if there are significant everyday cognitive difficulties, and especially if they are borne out by impairments on cognitive assessment, then interventions might be worth considering. 
Neuropsychology for DM1

Interventions could take several forms depending on the individual's insight and aspirations, the role of secondary factors including motor disability, medical prognosis, family support or stress, and the individual's cognitive profile as determined by neuropsychological testing. Intervention first involves ascertaining the key goals for the individual and his family. Next, one should consider the role of secondary factors such as fatigue and pain, and the extent to which they can be modified. Psychoeducation will include increasing the patient's (and possibly their family's and caregiver's) awareness of the individual's cognitive weaknesses and strengths. Compensatory methods may then be suggested to reduce the impact of cognitive impairments. For example, patients may be supported to make beneficial alterations to their home or work environment such as reducing clutter/proximity to distraction and keeping consistency in object placement or to use external aids such as smart-phones, notebooks, and diaries. Families, including the person with myotonic dystrophy, and carers may also get together to discuss how they can change how they interact in light of cognitive difficulties. Internal strategies such as mnemonics and visual imagery can be used to compensate for weakness in learning or retaining information(24). Of interest, there is also some, albeit lower-quality, evidence that more direct cognitive training could have some modest impact on working memory in multiple sclerosis (23), suggesting the possibility of a similar approach for people living with myotonic dystrophy type 1.

The work by Fujino et al. [4] suggests that clinicians working with myotonic dystrophy type 1 may be most often required to make suggestions for impairments in executive functioning, processing speed, and attention. Some compensatory recommendations might include goal management training approaches that have been successfully applied to improve executive functioning in people with neurological conditions (25) and the use of cueing systems to keep participants focused on tasks and adhere to rules (24). From our clinical experience, other potentially helpful rehabilitation techniques for disorders of executive functioning include problem solving training, encouraging patients to plan, break down tasks and monitor solutions. Also, metacognitive or self-instructional training may be useful as a means to counteract impulsivity. 
Neuropsychology for DM1

Finally, if mood or psycho-social factors appear to be significant contributors to cognitive or everyday functioning, then psychological interventions such as talking therapies may also be helpful (see Graham et al., (1) for an outline of these treatments).

\section{Conclusion}

The study by Fujino et al. [4] emphasises that cognitive impairment and behavioural changes are often apparent in severe myotonic dystrophy type 1 , and that such impairments are associated with quality of life. While there is a lack of empirical study of cognitive rehabilitation in myotonic dystrophy, given these findings, we suggest that clinical neuropsychology has a role in myotonic dystrophy care. Clinicians should be careful in choosing cognitive tests and measures of behaviour symptoms/behaviour change, as the muscle weakness and myotonia, fatigue, pain, depression and apathy may affect test scores. Yet, the characterisation of a cognitive and behavioural profiles may be beneficial and could lead to fruitful recommendations for intervention.

\section{List of abbreviations}

ALS = Amyotrophic Lateral Sclerosis

CTG = cytosine-thymine-guanine

D-KEFS = Delis Kaplan Executive Function Scale

DM1 = myotonic dystrophy type 1

RBANS = Repeatable Battery for the Assessment of Neurological Status WAIS = Wechsler Adult Intelligence Scale WMS $=$ Wechsler Memory Scale 
Neuropsychology for DM1

1. Graham CD, Simmons Z, Stuart SR, Rose MR. The potential of psychological interventions to improve quality of life and mood in muscle disorders. Muscle \& Nerve. $2015 ; 52(1): 131-6$.

2. Turner C, Hilton-Jones D. The myotonic dystrophies: diagnosis and management. Journal of Neurology, Neurosurgery \& Psychiatry. 2010;81(4):358.

3. Bird TD. Myotonic dystrophy type 1. [cited 2018 02/01/2018]. Available from: http://www.ncbi.nlm.nih.gov/books/NBK1165/.

4. Fujino $H$, Shingaki $H$, Suwazono S, Ueda $Y$, Wada C, Nakayama T, et al. Cognitive impairment and quality of life in patients with myotonic dystrophy type 1 . Muscle Nerve. 2017.

5. Sistiaga A, Urreta I, Jodar M, Cobo AM, Emparanza J, Otaegui D, et al. Cognitive/personality pattern and triplet expansion size in adult myotonic dystrophy type 1 (DM1): CTG repeats, cognition and personality in DM1. Psychological Medicine. 2009;40(3):487-95.

6. Winblad S, Lindberg C, Hansen S. Cognitive deficits and CTG repeat expansion size in classical myotonic dystrophy type 1 (DM1). Behavioral and Brain Functions. 2006;2:16-. 7. Gallais B, Montreuil M, Gargiulo M, Eymard B, Gagnon C, Laberge L. Prevalence and correlates of apathy in myotonic dystrophy type 1. BMC Neurology. 2015;15:148.

8. Winblad S, Hellstrom P, Lindberg C, Hansen S. Facial emotion recognition in myotonic dystrophy type 1 correlates with CTG repeat expansion. Journal of neurology, neurosurgery, and psychiatry. 2006;77(2):219-23.

9. Romeo V, Pegoraro E, Ferrati C, Squarzanti F, Soraru G, Palmieri A, et al. Brain involvement in myotonic dystrophies: neuroimaging and neuropsychological comparative study in DM1 and DM2. J Neurol. 2010;257(8):1246-55. 
Neuropsychology for DM1

10. Caso F, Agosta F, Peric S, Rakočević-Stojanović V, Copetti M, Kostic VS, et al. Cognitive Impairment in Myotonic Dystrophy Type 1 Is Associated with White Matter Damage. PLOS ONE. 2014;9(8):e104697.

11. Schneider-Gold C, Bellenberg B, Prehn C, Krogias C, Schneider R, Klein J, et al.

Cortical and Subcortical Grey and White Matter Atrophy in Myotonic Dystrophies Type 1 and 2 Is Associated with Cognitive Impairment, Depression and Daytime Sleepiness. PLOS ONE. 2015;10(6):e0130352.

12. Winblad S, Jensen C, Månsson J-E, Samuelsson L, Lindberg C. Depression in Myotonic Dystrophy type 1: clinical and neuronal correlates. Behavioral and Brain Functions : BBF. 2010;6:25-.

13. Randolph C, Tierney MC, Mohr E, Chase TN. The Repeatable Battery for the Assessment of Neuropsychological Status (RBANS): Preliminary Clinical Validity. Journal of Clinical and Experimental Neuropsychology. 1998;20(3):310-9.

14. Wechsler D. Wechsler adult intelligence scale-Fourth Edition (WAIS-IV). San Antonio, TX: NCS Pearson. 2008;22:498.

15. Wechsler D. Wechsler memory scale (WMS-III): Psychological corporation San Antonio, TX; 1997.

16. Delis DC, Kaplan E, Kramer JH. Delis-Kaplan executive function system (D-KEFS): Psychological Corporation; 2001.

17. Watt S, Crowe SF. Examining the beneficial effect of neuropsychological assessment on adult patient outcomes: a systematic review. The Clinical neuropsychologist. 2017:1-23. 18. Kobayakawa M, Tsuruya N, Kawamura M. Theory of mind impairment in adult-onset myotonic dystrophy type 1 . Neuroscience Research. 2012;72(4):341-6. 
Neuropsychology for DM1

19. Abrahams S, Newton J, Niven E, Foley J, Bak TH. Screening for cognition and behaviour changes in ALS. Amyotrophic lateral sclerosis \& frontotemporal degeneration. 2014;15(1-2):9-14.

20. Axford MM, Pearson CE. Illuminating CNS and cognitive issues in myotonic dystrophy: Workshop report. Neuromuscular Disorders. 2013;23(4):370-4.

21. Radakovic R, Abrahams S. Developing a new apathy measurement scale: dimensional apathy scale. Psychiatry research. 2014;219(3):658-63.

22. Wilson BA. Case studies in neuropsychological rehabilitation: Oxford University Press on Demand; 1999.

23. Rosti-Otajarvi EM, Hamalainen PI. Neuropsychological rehabilitation for multiple sclerosis. The Cochrane database of systematic reviews. 2014(2):Cd009131.

24. Fish J, Manly T, Emslie H, Evans JJ, Wilson BA. Compensatory strategies for acquired disorders of memory and planning: differential effects of a paging system for patients with brain injury of traumatic versus cerebrovascular aetiology. Journal of neurology, neurosurgery, and psychiatry. 2008;79(8):930-5.

25. Levine B, Schweizer TA, O'Connor C, Turner G, Gillingham S, Stuss DT, et al. Rehabilitation of Executive Functioning in Patients with Frontal Lobe Brain Damage with Goal Management Training. Frontiers in Human Neuroscience. 2011;5:9.

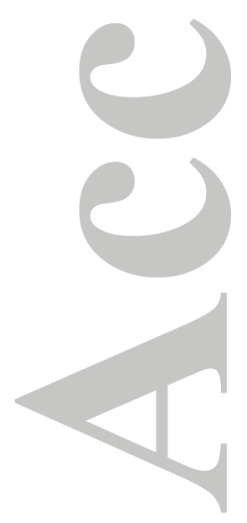

